\title{
Editorial
}

Nephrology

\section{Treatment and Outcome of Primary and Secondary Thrombotic Microangiopathies}

\author{
Ahmet Emre Eskazan Ayse Salihoglu \\ Division of Hematology, Department of Internal Medicine, Cerrahpasa Faculty of Medicine, Istanbul University, \\ Istanbul, Turkey
}

The first description of thrombotic microangiopathy (TMA), presumably thrombotic thrombocytopenic purpura (TTP), was by Moschcowitz in 1924 [1]. It was a disease presenting with a pentad consisting of anemia, thrombocytopenia, fever, hemiparesis and hematuria, and the postmortem evaluation showed widespread thrombi, mainly composed of platelets, in the terminal circulation of several organs. Although TTP was first characterized by the classical diagnostic pentad, it can present without all of these findings, and the presence of otherwise unexplained microangiopathic hemolytic anemia (MAHA) and thrombocytopenia strongly suggests TTP, which are considered 'sine qua non'.

Unusually large multimers of von Willebrand factor (vWF) were observed in patients with TTP [2], which then led to the discovery of a vWF-cleaving proteaseADAMTS13 [3]. ADAMTS13 cleaves vWF multimers that are secreted from vascular endothelial cells, and acquired TTP is an autoimmune disorder caused by inhibitory effects of autoantibodies on ADAMTS13 enzymatic activity.

In this issue of American Journal of Nephrology, Dahlan and colleagues shared their experience in 137 patients with primary and secondary TMAs, which were referred to their center for suspected TTP and received therapeutic plasma exchange (TPE) as a first-line treatment for over a decade [4]. The authors displayed the demographic data of the patients as well as the treatment modalities and outcomes.

The primary TMA syndromes can be either hereditary (including hereditary TTP (also called Upshaw - Schulman syndrome)) or acquired [5]. Acquired TMA syndromes

\section{KARGER 125}

(c) 2015 S. Karger AG, Basel

0250-8095/15/0416-0427\$39.50/0

E-Mail karger@karger.com

www.karger.com/ajn include classical ADAMTS13 deficient acquired (primaryidiopathic) TTP, Shiga toxin-mediated TMA (also called hemolytic-uremic syndrome (HUS)), drug-mediated TMA and complement-mediated TMA (so called atypical HUS). In addition to these, systemic infections, disseminated cancer, severe preeclampsia inc. Hemolysis, Elevated Liver enzymes, and Low Platelets (HELLP) syndrome, malignant hypertension, autoimmune disorders (e.g. systemic lupus erythematosus (SLE), systemic sclerosis (SS), catastrophic antiphospholipid syndrome (CAPS)) and hematopoietic stem-cell (HSCT) or organ transplantations are sometimes associated with TMA. Also, there is a distinct clinical entity resembling TTP occurring after surgical procedures, which is called 'postoperative TTP' (pTTP) [6].

In the cohort of Dahlan et al. [4], nearly half of the patients had no identifiable cause, whereas the other half were found to be with some medical conditions, mainly including infections, drug use, malignancies. Among the cases with secondary TMAs, 10 patients had confirmed HUS secondary to Shiga toxin-producing Escherichia coli O157:H7, of which nine fully recovered with TPE, whereas one died due to brain stem infarction.

Drug-induced TMA was identified among 17 patients, and 12 patients had an underlying malignancy (both hematological and solid tumors) [4]. In patients with druginduced TMA, nearly $30 \%$ of them died, whereas the remaining 12 survived, of which some fully recovered and some went into remission with renal and neurological sequelae. As expected, the mortality rate among malignancy-associated TMA was as high as $75 \%$. Connective tissue disease (mainly SLE, SS and CAPS) was identified in elev- 
en female patients, and 3 patients had a pregnancy-related TMA. Three patients developed PTTP, 2 developed TTP after HSCT, and 1 patient had TMA after pancreatitis.

In the historical data, the overall mortality of TTP was up to $90 \%$, but after the introduction of TPE, it has decreased to less than $30 \%$ [7]. However, a subset of patients with acquired TTP requires long-term plasma therapy to prevent fatal outcome and to achieve a sustained remission. Among these patients, immunosuppressive agents, high-dose intravenous immunoglobulin, rituximab, and splenectomy have been proposed [8]. Despite all these therapeutic options, however, recurrent acute episodes occur in approximately $40 \%$ of patients with acquired ADAMTS13 deficiency; most relapses occur within the first year [9]. The risk of recurrences are higher in patients with severe ADAMTS13 deficiency, and after the initial treatment with TPE, during the follow-up, persistence of ADAMTS13 deficiency together with increased concentrations of ADAMTS13 inhibitor is also associated with higher risk of recurrence [8].

The remission rate for patients with acquired idiopathic TTP in the presented cohort after TPE was $87 \%$, and approximately $75 \%$ of the patients maintained this response during a median follow-up of 73 months [4]. Since the ADAMTS13 activity, antigen and inhibitor concentrations were not tested neither at diagnosis nor during the follow-up, the authors could not display the follow-up data on that [4].

Among the entire patient cohort, the overall mortality rate was $23 \%$, and one fifth of these patients died while they were receiving TPE at the initial presentation. Putting primary and secondary cases together, nearly $60 \%$ of them were successfully treated with initial TPE therapy, while $20 \%$ remained refractory and received additional treatments. Among these refractory cases, various treat- ment options such as steroids, rituximab, other immunosuppressive agents, and splenectomy were used in addition to TPE, and also high-volume TPE was performed. The mortality rate for the refractory cases was $15 \%$. Nine patients experienced 12 relapses during the follow-up, of which one died but the others achieved remission after various lines of treatments [4].

The treatment option for acquired TTP is TPE, but although administered as an initial treatment as well, do patients with secondary TMAs really benefit from TPE? In general, patients with secondary TMA do not respond well to TPE, but there are some exceptions including various autoimmune conditions, pregnancy-related TMAs and drug-induced TMAs. In the cohort of Dahlan et al. [4], as the authors stated, the survival rate of $85 \%$ for the secondary causes of TMA suggests a possible benefit of TPE for these patients. In the secondary cases, the treatment focusing on the underlying etiology should be administered simultaneously as well.

Although ADAMTS13 assays are crucial and help to confirm the diagnosis of TTP, this is not always straightforward especially in developing countries, and physicians should not wait for the determination of ADAMTS13 activity to make the diagnosis of TTP, because this could take several days and could delay the initiation of TPE, which may result in morbidity and/or mortality. Thus, diagnostic work-up should be planned and TPE must be started simultaneously, but it should also be kept in mind that the response evaluation to TPE is of enormous importance for avoidance of the inappropriate use of TPE.

\section{Disclosure Statement}

All authors have no conflict of interest to declare.

\section{References}

1 Moschcowitz E: Hyaline thrombosis of the terminal arterioles and capillaries: a hitherto undescribed disease. Proc NY Pathol Soc 1924;24:21-24.

2 Moake JL, Rudy CK, Troll JH, et al: Unusually large plasma factor VIII: von Willebrand factor multimers in chronic relapsing thrombotic thrombocytopenic purpura. N Engl J Med 1982;307:1432-1435.

3 Levy GG, Nichols WC, Lian EC, et al: Mutations in a member of the ADAMTS gene family cause thrombotic thrombocytopenic purpura. Nature 2001;413:488494.
4 Dahlan R, Sontrop JM, Li L, et al: Primary and secondary thrombotic microangiopathy referred to a single plasma exchange centre for suspected thrombotic thrombocytopenic purpura: 2000-2011. Am J Nephrol 2015;41: 429-437.

5 George JN, Nester CM: Syndromes of thrombotic microangiopathy. N Engl J Med 2014; 371:1846-1848.

6 Eskazan AE, Buyuktas D, Soysal T: Postoperative thrombotic thrombocytopenic purpura. Surg Today 2015;45:8-16.

7 Bell WR, Braine HG, Ness PM, et al: Improved survival in thrombotic thrombocyto- penic purpura-hemolytic uremic syndrome. Clinical experience in 108 patients. N Engl J Med 1991;325:398-403.

8 Yilmaz M, Eskazan AE, Unsal A, et al: Cyclosporin A therapy on idiopathic thrombotic thrombocytopenic purpura in the relapse setting: two case reports and a review of the literature. Transfusion 2013;53:1586-1593.

9 George JN: The thrombotic thrombocytopenic purpura and hemolytic uremic syndromes: evaluation, management, and longterm outcomes experience of the Oklahoma TTP-HUS Registry, 1989-2007. Kidney Int Suppl 2009;112:S52-S54. 